\title{
Robust numerical method for singularly perturbed differential equations having both large and small delay
}

\author{
Habtamu Garoma Debela \\ College of Natural Sciences, Jimma University, Jimma, Ethiopia
}

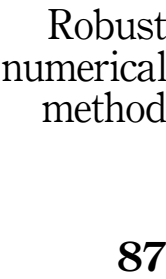

Received 7 September 2020 Revised 28 October 2020 Accepted 1 December 2020

\begin{abstract}
Purpose - The purpose of this study is to develop stable, convergent and accurate numerical method for solving singularly perturbed differential equations having both small and large delay.

Design/methodology/approach - This study introduces a fitted nonpolynomial spline method for singularly perturbed differential equations having both small and large delay. The numerical scheme is developed on uniform mesh using fitted operator in the given differential equation.

Findings - The stability of the developed numerical method is established and its uniform convergence is proved. To validate the applicability of the method, one model problem is considered for numerical experimentation for different values of the perturbation parameter and mesh points.

Originality/value - In this paper, the authors consider a new governing problem having both small delay on convection term and large delay. As far as the researchers' knowledge is considered numerical solution of singularly perturbed boundary value problem containing both small delay and large delay is first being considered.
\end{abstract}

Keywords Singularly perturbed, Small delay, Large delay, Fitted spline, $\varepsilon$-uniformly convergent

Paper type Research paper

\section{Introduction}

A differential equation is said to be singularly perturbed delay differential equation, if it includes at least one delay term, involving unknown functions occurring with different arguments, and also, the highest derivative term is multiplied by a small parameter. Such type of delay, differential equations play a very important role in the mathematical models of science and engineering, such as the human pupil light reflex with mixed delay type [1], variational problems in control theory with small state problem [2], models of HIV infection [3] and signal transition [4].

Any system involving a feedback control almost involves time delay. The delay occurs because a finite time is required to sense the information and then react to it. Finding the solution of singularly perturbed delay differential equations, whose application mentioned above, is a challenging problem. In response to these, in recent years, there has been a growing interest in numerical methods on singularly perturbed delay differential equations. The

\section{MSC - 65L11, 65L12, 65L20}

(C) Habtamu Garoma Debela. Published in Arab Journal of Mathematical Sciences. Published by Emerald Publishing Limited. This article is published under the Creative Commons Attribution (CC BY 4.0) licence. Anyone may reproduce, distribute, translate and create derivative works of this article (for both commercial and non-commercial purposes), subject to full attribution to the original publication and authors. The full terms of this licence may be seen at http://creativecommons.org/licences/by/4.0/ legalcode

The authors wish to express their thanks to Jimma University, College of Natural Sciences, for technical support and the authors of the literature for the provided scientific aspects and idea for this work.

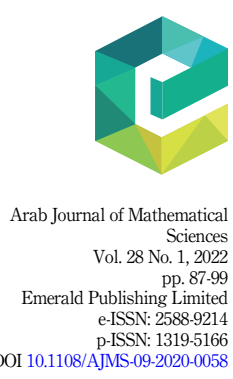


AJMS 28,1

88

authors of [5-7] have developed various numerical schemes on uniform meshes for singularly perturbed second-order differential equations having small delay on convection term. The authors of [8-12] have presented second-order differential equations having large delay.

In this paper, we consider a new governing problem having both small delay on convection term and large delay. Additionally, in recent years the correlative physical phenomena in-depth of the problem under consideration have been done by the authors [13-17]. As far as the researchers' knowledge is considered numerical solution of singularly perturbed boundary value problem containing both small delay and large delay is first being considered. Thus, the purpose of this study is to develop stable, convergent and accurate numerical method for solving singularly perturbed differential-difference equations having both small and large delay.

Throughout our analysis $C$ is generic positive constant that are independent of the parameter $\varepsilon$ and number of mesh points $2 N$. We assume that $\bar{\Omega}=[0,2], \Omega=(0,2)$, $\Omega_{1}=(0,1), \Omega_{2}=(1,2)$ and $\Omega^{*}=\Omega_{1} \cup \Omega_{2} . L_{1}$ and $L_{2}$ are the linear operator associated to the domain $\Omega_{1}$ and $\Omega_{2}$, respectively.

\section{Statement of the problem}

Consider the following singularly perturbed problem

$$
\begin{gathered}
L y(x)=-\varepsilon y^{\prime \prime}(x)+a(x) y^{\prime}(x)+b(x) y(x)+c(x) y(x-1)+d(x) y^{\prime}(x-\delta)=f(x), \\
y(x)=\phi(x), x \in[-1,0], y(2)=l,
\end{gathered}
$$

where $\delta$ is small, that is $\delta=O(\varepsilon), 0<\varepsilon \ll 1, \phi(x)$ is sufficiently smooth on [-1, 0]. For all $x \in \Omega$, it is assumed that the sufficient smooth functions $a(x), b(x), c(x)$ and $d(x)$ satisfy at $a(x) \geq a_{1}>a>0, b(x)>b \geq 0, c(x) \leq \gamma<0, d(x) \geq \zeta \geq 0$, and $2(a+\zeta)+5 b+5 \gamma>\eta>0$, $a\left(a_{1}-a\right)+2 \gamma>0$. The above assumptions ensure that $y \in X=C^{0}(\bar{\Omega}) \cap C^{1}(\Omega) \cap C^{2}\left(\Omega_{1} \cup \Omega_{2}\right)$.

The boundary value problem 1-2 exhibits strong boundary layer at $x=2$ and interior layer at $x=1$. Expand $y^{\prime}(x-\delta)$ about $x$ using the Taylor's expansion and discard higher order terms. Then, the above problem can be approximated by

$$
c_{\varepsilon, \delta}(x) y^{\prime \prime}(x)+p(x) y^{\prime}(x)+b(x) y(x)+c(x) y(x-1)=f(x),
$$

where $c_{\varepsilon, \delta}(x)=-\varepsilon-\delta d(x)$ and $p(x)=a(x)+d(x)$,

$$
y(x)=\phi(x), x \in[-1,0], y(2)=l .
$$

As we observed from Eqns (3) and (4), the values of $y(x-1)$ is known for the domain $\Omega_{1}$ and unknown for the domain $\Omega_{2}$ due to the large delay at $x=1$. So, it is impossible to treat the problem throughout the domain $(\bar{\Omega})$. Thus, we have to treat the problem at $\Omega_{1}$ and $\Omega_{2}$ separately. Eqns (3)-(4) are equivalent to

$$
L y(x)=R(x),
$$

where

$$
\begin{gathered}
L y(x)=\left\{\begin{array}{l}
L_{1} y(x)=c_{\varepsilon, \delta}(x) y^{\prime \prime}(x)+p(x) y^{\prime}(x)+b(x) y(x), x \in \Omega_{1}, \\
L_{2} y(x)=c_{\varepsilon, \delta}(x) y^{\prime \prime}(x)+p(x) y^{\prime}(x)+b(x) y(x)+c(x) y(x-1), \\
x \in \Omega_{2} .
\end{array}\right. \\
R(x)=\left\{\begin{array}{l}
f(x)-c(x) \phi(x-1), x \in \Omega_{1}, \\
f(x), x \in \Omega_{2} .
\end{array}\right.
\end{gathered}
$$


with boundary conditions

Robust

$$
\left\{\begin{array}{l}
y(x)=\phi(x), x \in[-1,0] \\
y\left(1^{-}\right)=y\left(1^{+}\right), y^{\prime}\left(1^{-}\right)=y^{\prime}\left(1^{+}\right), \\
y(2)=l
\end{array}\right.
$$

\section{Properties of continuous solution}

Lemma 3.1. (Maximum principle) Let $\psi(x)$ be any function in $X$ such that $\psi(0) \geq 0$, $\psi(2) \geq 0, L_{1} \psi(x) \geq 0, \forall x \in \Omega_{1}, L_{2} \psi(x) \geq 0, \forall x \in \Omega_{2}$ and $\left[\psi^{\prime}\right](1) \leq 0$ then $\psi(x) \geq 0, \forall x \in \bar{\Omega}$

Proof. For the proof refer [8]

Lemma 3.2. (Stability result) The solution $y(x)$ of problem (3)-(4), satisfies the bound

$$
|y(x)| \leq C \max \left\{|y(0)|,|y(2)|, \sup _{x \in \Omega^{*}}|L y(x)|\right\}, x \in \bar{\Omega} .
$$

Proof. For the proof refer [8]

Lemma 3.3. Let $y(x)$ be the solution of (3)-(4). Then we have the following bounds

$$
\left\|y^{(k)}(x)\right\|_{\Omega^{*}} \leq C \varepsilon^{-k}, \quad k=1,2,3 .
$$

Proof. For the proof refer [8]

\section{Numerical scheme formulation}

We divide the interval $[0,2]$ into $2 N$ equal parts with constant mesh length $h$. Let $0=x_{0}, x_{1}, \ldots, x_{N}=1, x_{N+1}, x_{N+2}, \ldots, x_{2 N}=2$ be the mesh points. Then we have $x_{i}=i h, i=0,1,2, \ldots, 2 N$.

Consider a uniform mesh with interval $[0,1]$ in which $0=x_{0}<x_{1}<\ldots<x_{N}=1$ where $h=\frac{1}{N}$ and $x_{i}=i h, i=0,1,2, \ldots, N$.

We can rewrite (3) as

$$
c_{\varepsilon, \delta}(x) y^{\prime \prime}(x)+p(x) y^{\prime}(x)+b(x) y(x)=Q(x), x \in \Omega_{1},
$$

where $Q(x)=f(x)-c(x) y(x-1)$.

For each segment $\left[x_{i}, x_{i+1}\right], i=1,2, \ldots, N-1$ the non-polynomial cubic spline $S_{\Delta}(x)$ has the following form

$$
S_{\Delta}(x)=a_{i}+b_{i}\left(x-x_{i}\right)+c_{i}\left(e^{w\left(x-x_{i}\right)}+e^{-w\left(x-x_{i}\right)}\right)+d_{i}\left(e^{w\left(x-x_{i}\right)}-e^{-w\left(x-x_{i}\right)}\right),
$$

where $a_{i}, b_{i}, c_{i}$ and $d_{i}$ are unknown coefficients, and $w \neq 0$ arbitrary parameter which will be used to increase the accuracy of the method.

To determine the unknown coefficients in (10) in terms of $y_{i}, y_{i+1}, M_{i}$ and $M_{i+1}$ first we define

$$
\begin{cases}S_{\Delta}\left(x_{i}\right)=y_{i}, & S_{\Delta}\left(x_{i+1}\right)=y_{i+1}, \\ S_{\Delta}^{\prime \prime}\left(x_{i}\right)=M_{i}, & S_{\Delta}^{\prime \prime}\left(x_{i+1}\right)=M_{i+1} .\end{cases}
$$


AJMS

28,1

90

The coefficients in (10) are determined as

where $\theta=w h$.

$$
\left\{\begin{aligned}
a_{i} & =y_{i}-\frac{M_{i}}{w^{2}}, \\
b_{i} & =\frac{y_{i+1}-y_{i}}{h}+\frac{M_{i}-M_{i+1}}{w \theta}, \\
c_{i} & =\frac{M_{i+1}}{w^{2}\left(e^{\theta}-e^{-\theta}\right)}-\frac{M_{i}\left(e^{\theta}+e^{-\theta}\right)}{2 w^{2}\left(e^{\theta}-e^{-\theta}\right)}, \\
d_{i} & =\frac{M_{i}}{2 w^{2}},
\end{aligned}\right.
$$

Reducing indices of Eqn (12) by one and substituting into Eqn (13), we obtain $\frac{y_{i}-y_{i-1}}{h}+\frac{M_{i}-M_{i+1}}{w \theta}+w\left(\frac{2 M_{i}-\left(e^{\theta}+e^{-\theta}\right) M_{i-1}}{2 w^{2}\left(e^{\theta}+e^{-\theta}\right)}\right)=\frac{y_{i+1}-y_{i}}{h}+\frac{M_{i}-M_{i+1}}{w \theta}+2 w\left(\frac{M_{i+1}}{w^{2}\left(e^{\theta}-e^{-\theta}\right)}-\frac{M_{i}\left(e^{\theta}+e^{-\theta}\right)}{2 w^{2}\left(e^{\theta}-e^{-\theta}\right)}\right)$,

$$
\Rightarrow \frac{y_{i-1}-2 y_{i}+y_{i+1}}{h^{2}}=\alpha M_{i-1}+2 \beta M_{i}+\alpha M_{i+1},
$$

where

$$
\alpha=\frac{1}{\theta^{2}}\left(1-\frac{2 \theta}{\left(e^{\theta}-e^{-\theta}\right)}\right), \beta=\frac{1}{\theta^{2}}\left(\frac{\theta\left(e^{\theta}+e^{-\theta}\right)}{\left(e^{\theta}-e^{-\theta}\right)}-1\right) .
$$

If $h \rightarrow 0$, then $\theta=h k \rightarrow 0$. Thus, using L'Hopitals rule we have $\lim _{h \rightarrow 0} \alpha=\frac{1}{6}$ and $\lim _{h \rightarrow 0} \beta=\frac{1}{3}$.

Using $S_{\Delta}^{\prime \prime}\left(x_{i}\right)=y_{i}^{\prime \prime}=M_{i}$ in to (9), we get

$$
\left\{\begin{array}{l}
c_{\varepsilon, \delta}(x) M_{i}=Q_{i}-p_{i} y_{i}^{\prime}-b_{i} y_{i}, \\
c_{\varepsilon, \delta}(x) M_{i-1}=Q_{i-1}-p_{i-1} y_{i-1}^{\prime}-b_{i-1} y_{i-1}, \\
c_{\varepsilon, \delta}(x) M_{i+1}=Q_{i+1}-p_{i+1} y_{i+1}^{\prime}-b_{i+1} y_{i+1} .
\end{array}\right.
$$

Using Taylorâ ${ }^{\mathrm{TM}_{\mathrm{S}}}$ series expansions of $y_{i-1}, y_{i+1}, y_{i-1}^{\prime}$ and $y_{i+1}^{\prime}$ simplifying, we have

$$
\left\{\begin{array}{l}
y_{i}^{\prime}=\frac{y_{i+1}-y_{i-1}}{2 h}+T_{1}, \\
y_{i-1}^{\prime}=\frac{-y_{i+1}+4 y_{i}-3 y_{i-1}}{2 h}+T_{2}, \\
y_{i+1}^{\prime}=\frac{3 y_{i+1}-4 y_{i}+y_{i-1}}{2 h}+T_{2},
\end{array}\right.
$$

where

$$
T_{1}=-\frac{h^{2}}{6} y^{\prime \prime \prime}(\xi) \text { and } T_{2}=\frac{h^{2}}{12} y^{\prime \prime \prime}(\xi) \text {, for } \xi \in\left(x_{i-1}, x_{i}\right) .
$$


Substituting Eqn (16) in to Eqn (15), we obtain

Robust

$$
\left\{\begin{array}{l}
M_{i}=\frac{1}{c_{\varepsilon, \delta}(x)}\left\{Q_{i}-p_{i}\left(\frac{y_{i+1}-y_{i-1}}{2 h}+T_{1}\right)-b_{i} y_{i}\right\}, \\
M_{i-1}=\frac{1}{c_{\varepsilon, \delta}(x)}\left\{Q_{i-1}-p_{i-1}\left(\frac{-y_{i+1}+4 y_{i}-3 y_{i-1}}{2 h}+T_{2}\right)-b_{i-1} y_{i-1}\right\}, \\
M_{i+1}=\frac{1}{c_{\varepsilon, \delta}(x)}\left\{Q_{i+1}-p_{i+1}\left(\frac{3 y_{i+1}-4 y_{i}+y_{i-1}}{2 h}+T_{2}\right)-b_{i+1} y_{i+1}\right\} .
\end{array}\right.
$$

Substituting Eqn (17) into Eqn (14) and rearranging, we get

$$
\begin{aligned}
& \frac{c_{\varepsilon, \delta}(x)}{h^{2}}\left(y_{i-1}-2 y_{i}+y_{i+1}\right)+\frac{\alpha p_{i-1}}{2 h}\left(-y_{i+1}-4 y_{i}-3 y_{i-1}\right)+\frac{2 \beta p_{i}}{2 h}\left(y_{i+1}-y_{i-1}\right) \\
& \quad+\frac{\alpha p_{i+1}}{2 h}\left(3 y_{i+1}-3 y_{i}+y_{i-1}\right)=\alpha\left(Q_{i-1}-b_{i-1} y_{i-1}+Q_{i+1}-b_{i+1} y_{i+1}\right) \\
& \quad+2 \beta\left(Q_{i}-b_{i} y_{i}\right)+T,
\end{aligned}
$$

where, $T=\left(4 \beta p_{i}-\alpha p_{i-1}-\alpha p_{i+1}\right) \frac{h^{2}}{12} y^{\prime \prime \prime}(\xi)$ is the local truncation error.

From the theory of singular perturbations described in [18] and the Taylorâ ${ }^{\mathrm{TM}_{\mathrm{S}}}$ series expansion of $y(x)$ about the point ' 0 ' in the asymptotic solution of the problem in Eq. (9), we have

$$
y\left(x_{i}\right) \approx y_{0}\left(x_{i}\right)+\left(\phi_{0}-y_{0}(0)\right) e^{-p(0) \frac{i h}{\varepsilon_{\varepsilon, \delta}(x)}}
$$

and letting $\rho=\frac{h}{c_{\varepsilon, \delta}(x)}$, we get

$$
\lim _{h \rightarrow 0} y(i h) \approx y_{0}(i h)+\left(\phi_{0}-y_{0}(0)\right) e^{-p(0) i \rho},
$$

since $x_{i}=x_{0}+i h$.

Introducing a fitting factor $\sigma(\rho)$ in to Eq. (18), we get

$$
\begin{aligned}
\frac{\sigma(\rho) c_{\varepsilon, \delta}(x)}{h^{2}} & \left(y_{i-1}-2 y_{i}+y_{i+1}\right)+\frac{\alpha p_{i-1}}{2 h}\left(-y_{i+1}-4 y_{i}-3 y_{i-1}\right)+\frac{2 \beta p_{i}}{2 h}\left(y_{i+1}-y_{i-1}\right) \\
& +\frac{\alpha p_{i+1}}{2 h}\left(3 y_{i+1}-3 y_{i}+y_{i-1}\right)=\alpha\left(Q_{i-1}-b_{i-1} y_{i-1}+Q_{i+1}-b_{i+1} y_{i+1}\right) \\
& +2 \beta\left(Q_{i}-b_{i} y_{i}\right)+T .
\end{aligned}
$$

Multiplying Eqn (19) by $h$ and taking a limit as $h \rightarrow 0$ we get

$$
\begin{aligned}
\frac{\sigma}{\rho} \lim _{h \rightarrow 0}\left(y_{i-1}\right. & \left.-2 y_{i}+y_{i+1}\right)+\frac{\alpha p(0)}{h} \lim _{h \rightarrow 0}\left(-y_{i+1}-4 y_{i}-3 y_{i-1}\right) \\
& +\beta p(0) \lim _{h \rightarrow 0}\left(y_{i+1}-y_{i-1}\right)+\frac{\alpha p(0)}{2} \lim _{h \rightarrow 0}\left(3 y_{i+1}-3 y_{i}+y_{i-1}\right)=0 .
\end{aligned}
$$

Thus, we consider two cases of the boundary layers. 
AJMS

28,1

92

Case 1: For $p(x)>0$ (Left-end boundary layer), we have

$$
\left\{\begin{array}{l}
\lim _{h \rightarrow 0}\left(y_{i-1}-2 y_{i}+y_{i+1}\right)=\left(\phi_{0}-y_{0}(0)\right) e^{-p(0) i \rho}\left(e^{p(0) \rho}+e^{-p(0) \rho}-2\right), \\
\lim _{h \rightarrow 0}\left(-y_{i+1}-4 y_{i}-3 y_{i-1}\right)=\left(\phi_{0}-y_{0}(0)\right) e^{-p(0) i \rho}\left(-3 e^{p(0) \rho}-e^{-p(0) \rho}+4\right), \\
\lim _{h \rightarrow 0}\left(y_{i+1}-y_{i-1}\right)=\left(\phi_{0}-y_{0}(0)\right) e^{-p(0) i \rho}\left(e^{p(0) \rho}+3 e^{-p(0) \rho}-4\right), \\
\lim _{h \rightarrow 0}\left(3 y_{i+1}-3 y_{i}+y_{i-1}\right)=\left(\phi_{0}-y_{0}(0)\right) e^{-p(0) i \rho}\left(e^{-p(0) \rho}-e^{p(0) \rho}\right) .
\end{array}\right.
$$

Substituting Eqn (21) into Eqn (20) and simplifying, we get

$$
\sigma_{0}=\rho p(0)(\alpha+\beta) \operatorname{coth}\left(\frac{p(0) \rho}{2}\right) .
$$

Case 2: For $p(x)<0$ (Right-end boundary layer), we have

$$
\left\{\begin{array}{l}
\lim _{h \rightarrow 0}\left(y_{i-1}-2 y_{i}+y_{i+1}\right)=\left(\varphi-y_{0}(1)\right) e^{-p(1) i \rho}\left(e^{p(1) \rho}+e^{-p(1) \rho}-2\right), \\
\lim _{h \rightarrow 0}\left(-y_{i+1}-4 y_{i}-3 y_{i-1}\right)=\left(\varphi-y_{0}(1)\right) e^{-p(1) \rho}\left(-3 e^{p(1) \rho}-e^{-p(1) \rho}+4\right), \\
\lim _{h \rightarrow 0}\left(y_{i+1}-y_{i-1}\right)=\left(\varphi-y_{0}(1)\right) e^{-p(1) i \rho}\left(e^{p(1) \rho}+3 e^{-p(1) \rho}-4\right), \\
\lim _{h \rightarrow 0}\left(3 y_{i+1}-3 y_{i}+y_{i-1}\right)=\left(\varphi-y_{0}(1)\right) e^{-p(1) i \rho}\left(e^{-p(1) \rho}-e^{p(1) \rho}\right) .
\end{array}\right.
$$

Substituting Eq. (22) into Eq. (20) and simplifying, we obtain

$$
\sigma_{1}=\rho p(1)(\alpha+\beta) \operatorname{coth}\left(\frac{p(1) \rho}{2}\right) .
$$

In general, we take a variable fitting parameter as

$$
\sigma_{i}=\rho_{i} p\left(x_{i}\right)(\alpha+\beta) \operatorname{coth}\left(\frac{p\left(x_{i}\right) \rho_{i}}{2}\right),
$$

where, $\rho_{i}=\frac{h}{c_{e, \delta}(x)}$.

Thus, Eqn (19) can be written as

$$
\begin{aligned}
\left\{\frac{\varepsilon_{\varepsilon, \delta}(x) \sigma_{i}}{h^{2}}\right. & \left.-\frac{3 \alpha p_{i-1}}{2 h}+\alpha b_{i-1}-\frac{\beta p_{i}}{h}+\frac{\alpha p_{i+1}}{2 h}\right\} y_{i-1}-\left\{\frac{2 c_{\varepsilon, \delta}(x) \sigma_{i}}{h^{2}}-\frac{2 \alpha p_{i-1}}{h}-2 \beta b_{i}+\frac{2 \alpha p_{i+1}}{h}\right\} y_{i} \\
& +\left\{\frac{c_{\varepsilon, \delta}(x) \sigma_{i}}{h^{2}}-\frac{\alpha p_{i-1}}{2 h}+\alpha b_{i+1}+\frac{\beta p_{i}}{h}+\frac{3 \alpha p_{i+1}}{2 h}\right\} y_{i+1} \\
= & \alpha\left(Q_{i-1}+Q_{i+1}\right)+2 \beta Q_{i} .
\end{aligned}
$$

Simplifying Eqn (25), for the domain $\Omega_{1}=(0,1)$, we get the tri-diagonal system of the equation of the form

$$
L^{N} \equiv E_{i} y_{i-1}-F_{i} y_{i}+G_{i} y_{i+1}=H_{i}, \quad i=1,2, \ldots, N-1,
$$


where

$$
\left\{\begin{array}{l}
E_{i}=\frac{c_{\varepsilon, \delta}(x) \sigma_{i}}{h^{2}}-\frac{3 \alpha p_{i-1}}{2 h}+\alpha b_{i-1}-\frac{\beta p_{i}}{h}+\frac{\alpha p_{i+1}}{2 h} \\
F_{i}=\frac{2 c_{\varepsilon, \delta}(x) \sigma_{i}}{h^{2}}-\frac{2 \alpha p_{i-1}}{h}-2 \beta b_{i}+\frac{2 \alpha p_{i+1}}{h} \\
G_{i}=\frac{c_{\varepsilon, \delta}(x) \sigma_{i}}{h^{2}}-\frac{\alpha p_{i-1}}{2 h}+\alpha b_{i+1}+\frac{\beta p_{i}}{h}+\frac{3 \alpha p_{i+1}}{2 h} \\
H_{i}=\alpha\left(Q_{i-1}+Q_{i+1}\right)+2 \beta Q_{i} .
\end{array}\right.
$$

Similarly, if we consider the domain $\Omega_{2}=(1,2)$, from Eqs. (3), we have

$$
\left\{\begin{array}{c}
c_{\varepsilon, \delta}(x) y^{\prime \prime}(x)+p(x) y^{\prime}(x)+b(x) y(x)+c(x) y(x-1)=f(x), \quad x \in \Omega_{2} \\
y(1)=\theta, \quad y(2)=l .
\end{array}\right.
$$

Using $S_{\Delta}^{\prime \prime}\left(x_{i}\right)=y_{i}^{\prime \prime}=M_{i}$ for Eqn (27), we get

$$
\left\{\begin{array}{l}
c_{\varepsilon, \delta}\left(x_{i}\right) M_{i}=f_{i}-p_{i} y_{i}^{\prime}-b_{i} y_{i}-c_{i} y\left(x_{i}-1\right), \\
c_{\varepsilon, \delta}\left(x_{i}\right) M_{i-1}=f_{i-1}-p_{i-1} y_{i-1}^{\prime}-b_{i-1} y_{i-1}-c_{i-1} y\left(x_{i-1}-1\right), \\
c_{\varepsilon, \delta}\left(x_{i}\right) M_{i+1}=f_{i+1}-p_{i+1} y_{i+1}^{\prime}-b_{i+1} y_{i+1}-c_{i+1} y\left(x_{i+1}-1\right) .
\end{array}\right.
$$

Substituting Eq. (16) in to Eq. (28), we obtain

$$
\left\{\begin{array}{l}
M_{i}=\frac{1}{c_{\varepsilon, \delta}\left(x_{i}\right)}\left\{f_{i}-p_{i}\left(\frac{y_{i+1}-y_{i-1}}{2 h}+T_{1}\right)-b_{i} y_{i}-c_{i} y\left(x_{i}-1\right)\right\} \\
M_{i-1}=\frac{1}{c_{\varepsilon, \delta}\left(x_{i}\right)}\left\{f_{i-1}-p_{i-1}\left(\frac{-y_{i+1}+4 y_{i}-3 y_{i-1}}{2 h}+T_{2}\right)-b_{i-1} y_{i-1}-c_{i-1} y\left(x_{i-1}-1\right)\right\} \\
M_{i+1}=\frac{1}{c_{\varepsilon, \delta}\left(x_{i}\right)}\left\{f_{i+1}-p_{i+1}\left(\frac{3 y_{i+1}-4 y_{i}+y_{i-1}}{2 h}+T_{2}\right)-b_{i+1} y_{i+1}-c_{i+1} y\left(x_{i+1}-1\right)\right\}
\end{array}\right.
$$

Substituting Eqn (29) in to Eqn (14), introducing fitting factor and rearranging, we get

$$
L^{N} \equiv E_{i} y_{i-1}-F_{i} y_{i}+G_{i} y_{i+1}+T_{i}=H_{i}, \quad i=N+1, N+2, \ldots, 2 N-1,
$$

where

$$
\left\{\begin{array}{l}
E_{i}=\frac{c_{\varepsilon, \delta}\left(x_{i}\right) \sigma_{i}}{h^{2}}-\frac{3 \alpha p_{i-1}}{2 h}+\alpha b_{i-1}-\frac{\beta p_{i}}{h}+\frac{\alpha p_{i+1}}{2 h}, \\
F_{i}=\frac{2 c_{\varepsilon, \delta}\left(x_{i}\right) \sigma_{i}}{h^{2}}-\frac{2 \alpha p_{i-1}}{h}-2 \beta b_{i}+\frac{2 \alpha p_{i+1}}{h} \\
G_{i}=\frac{c_{\varepsilon, \delta}\left(x_{i}\right) \sigma_{i}}{h^{2}}-\frac{\alpha p_{i-1}}{2 h}+\alpha b_{i+1}+\frac{\beta p_{i}}{h}+\frac{3 \alpha p_{i+1}}{2 h} \\
H_{i}=\alpha\left(f_{i-1}+f_{i+1}\right)+2 \beta f_{i}, \\
T_{i}=\alpha\left\{c_{i-1} y\left(x_{i-1}-1\right)+c_{i+1} y\left(x_{i+1}-1\right)+2 \beta c_{i} y\left(x_{i}-1\right)\right\}
\end{array}\right.
$$

Therefore, on the whole domain $\bar{\Omega}=[0,2]$, the basic schemes to solve Eqs (1)-(2) are the schemes given in Eqn (26) and Eqn (30). 
AJMS

28,1

94

\section{Stability and convergence analysis}

\subsection{Truncation error}

Let expand the terms $y_{i \pm 1}$ and $M_{i \pm 1}$ from Eqn (14), using Taylor's series as

$$
\left\{\begin{array}{l}
y_{i+1}=y_{i}+h y_{i}^{\prime}+\frac{h^{2}}{2 !} y_{i}^{\prime \prime}+\frac{h^{3}}{3 !} y_{i}^{\prime \prime \prime}+\frac{h^{4}}{4 !} y_{i}^{(4)}+\frac{h^{5}}{5 !} y_{i}^{(5)}+\frac{h^{6}}{6 !} y_{i}^{(6)}+O\left(h^{7}\right), \\
y_{i-1}=y_{i}-h y_{i}^{\prime}+\frac{h^{2}}{2 !} y_{i}^{\prime \prime}-\frac{h^{3}}{3 !} y_{i}^{\prime \prime \prime}+\frac{h^{4}}{4 !} y_{i}^{(4)}-\frac{h^{5}}{5 !} y_{i}^{(5)}+\frac{h^{6}}{6 !} y_{i}^{(6)}+O\left(h^{7}\right), \\
M_{i+1}=y_{i+1}^{\prime \prime}=y_{i}^{\prime \prime}+h y_{i}^{\prime \prime \prime}+\frac{h^{2}}{2 !} y_{i}^{(4)}+\frac{h^{3}}{3 !} y_{i}^{(5)}+\frac{h^{4}}{4 !} y_{i}^{(6)}+O\left(h^{7}\right), \\
M_{i-1}=y_{i-1}^{\prime \prime}=y_{i}^{\prime \prime}-h y_{i}^{\prime \prime \prime}+\frac{h^{2}}{2 !} y_{i}^{(4)}-\frac{h^{3}}{3 !} y_{i}^{(5)}+\frac{h^{4}}{4 !} y_{i}^{(6)}+O\left(h^{7}\right) .
\end{array}\right.
$$

The local truncation error $T_{i}(h)$ obtained from Eqn (14) as

$$
T_{i}(h)=\frac{y_{i-1}-2 y_{i}+y_{i+1}}{h^{2}}-\alpha\left(M_{i-1}+M_{i+1}\right)-2 \beta M_{i} .
$$

Substituting the series of $y_{i \pm 1}$ and $M_{i \pm 1}$ from Eqn (31)-(32) and collecting like terms gives

$$
T_{i}(h)=(1-2(\alpha+\beta)) y_{i}^{\prime \prime}+h^{2}\left(\frac{1}{12}-\alpha\right) y_{i}^{(4)}+O\left(h^{4}\right) .
$$

But from the values of $\alpha=\frac{1}{6}$ and $\beta=\frac{1}{3}$, Eqn (33) becomes

$$
T_{i}(h)=h^{2}\left(-\frac{1}{12}\right) y_{i}^{(4)}+O\left(h^{4}\right)
$$

which implies

$$
\left\|T_{i}(h)\right\| \leq C h^{2},
$$

where $C=\frac{1}{12}\left|y_{i}^{(4)}\right|$.

This establishes that the developed method is second order accurate or its order of convergence is $O\left(h^{2}\right)$.

\subsection{Convergence analysis}

Local truncation errors refer to the differences between the original differential equation and its finite difference approximation at a mesh points. Finite difference scheme is called consistent if the limit of truncation error $\left(T_{i}(h)\right)$ is equal to zero as the mesh size $h$ goes to zero. Hence, the proposed method in Eqn (26) with local truncation error in Eqn (34) satisfies the definition of consistency as

$$
\lim _{h \rightarrow 0} T_{i}(h)=\lim _{h \rightarrow 0} C h^{2}=0 .
$$

Thus, the proposed scheme is consistent. 
5.3 Stability analysis

Consider the developed scheme in Eqn (26),

$$
E_{i} y_{i-1}-F_{i} y_{i}+G_{i} y_{i+1}=H_{i}
$$

Robust numerical method

where the coefficients $E_{i}, F_{i}$ and $G_{i}$ are as in Eqn (26). If we multiply both sides of Eqn (26) by $h^{2}$ and consider the values of $E_{i}, F_{i}$ and $G_{i}$ for sufficiently small $h$, we get

$$
E_{i}=G_{i}=\varepsilon \sigma, F_{i}=2 \varepsilon \sigma,
$$

Considering Eqn (37) into Eqn (26) the one which is multiplied by $h^{2}$ the developed scheme can be written in a matrix form

$$
A Y=B
$$

where the matrices $A=\left(\begin{array}{ccccc}-2 \varepsilon \sigma & \varepsilon \sigma & 0 & \ldots & 0 \\ \varepsilon \sigma & -2 \varepsilon \sigma & \varepsilon \sigma & \ldots & 0 \\ 0 & - & - & & 0 \\ \vdots & & & & \varepsilon \sigma \\ 0 & - & - & \varepsilon \sigma & -2 \varepsilon \sigma\end{array}\right), Y=\left(\begin{array}{c}y_{1} \\ y_{2} \\ \vdots \\ y_{N-2} \\ y_{N-1}\end{array}\right)$ and

$$
B=\left(\begin{array}{c}
h^{2} H_{1}-E_{1} y_{0} \\
h^{2} H_{2} \\
\vdots \\
h^{2} H_{N-2} \\
h^{2} H_{N-1}-G_{N-1} y_{N}
\end{array}\right)
$$

Here, the coefficient matrix $A$ is a tri-diagonal matrix with size $(N-1) \times(N-1)$. Matrix $A$ is irreducible if its codiagonals contain nonzero elements only. The codiagonals contain $E_{i}$ and $G_{i}$. It is clearly seen that, for sufficiently small $h$ both $E_{i} \neq 0$ and $G_{i} \neq 0$ for $i=1,2, \ldots, N-1$. Hence, $A$ is irreducible.

Again we can see that all $\left|E_{i}\right|,|F|_{i},\left|G_{i}\right|>0$ for $i=1,2, \ldots, N-1$ and in each row of $\mathrm{A}$, the modulus of diagonal element is greater than or equal to the sum of the modulus of the two codiagonal elements (i.e. $\left.\left|F_{i}\right| \geq\left|E_{i}\right|+\left|G_{i}\right|\right)$. This implies that $A$ is diagonally dominant. Under this condition, the Thomas algorithm is stable for sufficiently small $h$.

As discussed in [19] the eigenvalues of a tri-diagonal matrix $A$ are given by

$$
\lambda_{s}=-2 \varepsilon \sigma+2\{\sqrt{(\varepsilon \sigma)(\varepsilon \sigma)}\} \cos \frac{s \pi}{N}, s=1(1) N-1 .
$$

Hence, the eigenvalues of matrix $A$ in Eqn (38) are

$$
\lambda_{s}=-2 \varepsilon \sigma+2\left\{\sqrt{(\varepsilon \sigma)^{2}}\right\} \cos \frac{s \pi}{N}=-2 \varepsilon \sigma\left(1-\cos \frac{s \pi}{N}\right), s=1(1) N-1 .
$$

But from trigonometric identity, we have $1-\cos \frac{s \pi}{N}=2 \sin ^{2} \frac{s \pi}{2 N}$. Thus, the eigenvalues of $A$

$$
\lambda_{s}=-2 \varepsilon \sigma\left(2 \sin ^{2} \frac{s \pi}{2 N}\right)=-4 \varepsilon \sigma \sin ^{2} \frac{s \pi}{2 N} \leq-4 \varepsilon \sigma .
$$


AJMS

28,1

96

A finite difference method for the boundary value problems is stable if $A$ is nonsingular and $\left\|A^{-1}\right\| \leq C$, for $0<h<h_{0}$. where, $C$ and $h_{0}$ are two constants that are independent of $h$.

Since $A$ is real and symmetric it follows that $A^{-1}$ is also real and symmetric so that, its eigenvalues are real and given by $\frac{1}{\lambda_{s}}$. Hence, as [19] the stability condition of the method will be satisfied when; ||$A^{-1} \|=\left|\frac{1}{\lambda_{s}}\right|=\left|\frac{-1}{4 \varepsilon \sigma}\right|=\frac{1}{4 \varepsilon \sigma} \leq C$, where, $C$ is independent of $h$. Thus the developed scheme in Eqn (26) is stable. A consistent and stable finite difference method is convergent by [20]. Hence as we have shown above, the proposed method is satisfying both the criteria of consistency and stability which are equivalent to convergence of the method.

\section{Numerical examples and results}

In this section, one example is given to illustrate the numerical method discussed above. The exact solutions of the test problem is not known. Therefore, we use the double mesh principle to estimate the error and compute the experiment rate of convergence to the computed solution. For this we put

$$
E_{\varepsilon}^{N}=\max _{0 \leq i \leq 2 N}\left|Y_{i}^{N}-Y_{2 i}^{2 N}\right|
$$

where $Y_{i}^{N}$ and $Y_{2 i}^{2 N}$ are the $i^{t h}$ components of the numerical solutions on meshes of $N$ and $2 N$, respectively. We compute the uniform error and the rate of convergence as

$$
E^{N}=\max _{\varepsilon} E_{\varepsilon}^{N} \text {, and } R^{N}=\log _{2}\left(\frac{E^{N}}{E^{2 N}}\right) .
$$

The numerical results are presented for the values of the perturbation parameter $\varepsilon \in\left\{10^{-4}, 10^{-8}, \ldots, 10^{-20}\right\}$.

Example 6.1. Consider the model singularly perturbed boundary value problem:

$$
-\varepsilon y^{\prime \prime}(x)+10 y^{\prime}(x)-y(x-1)+y^{\prime}(x-\varepsilon)=x x \in(0,1) \cup(1,2),
$$

Subject to the boundary conditions

$$
y(x)=1, x \in[-1,0], y(2)=2 .
$$

\section{Discussion and conclusion}

This study introduces a fitted nonpolynomial spline method for singularly perturbed differential equations having both small and large delay. The numerical scheme is developed on uniform mesh using fitted operator in the given differential equation. The stability of the developed numerical method is established and its uniform convergence is proved. To validate the applicability of the method, one model problem is considered for numerical experimentation for different values of the perturbation parameter and mesh points. The numerical results are tabulated in terms of maximum absolute errors, numerical rate of convergence and uniform errors (see Table 1). Further, behavior of the numerical solution (Figure 1), point-wise absolute error (Figure 2) and the $\varepsilon$-uniform convergence of the method is shown by the log-log plot (Figure 3). The method is shown to be $\varepsilon$-uniformly convergent 
with order of convergence $O\left(h^{2}\right)$. The proposed method gives accurate, stable and $\varepsilon$-uniform numerical result.

\section{Robust \\ numerical \\ method}

\begin{tabular}{|c|c|c|c|c|c|c|}
\hline$\varepsilon$ & $N=32$ & $N=64$ & $N=128$ & $N=256$ & $N=512$ & 97 \\
\hline $10^{-4}$ & $1.9799 \mathrm{e}-04$ & $1.0004 \mathrm{e}-04$ & $5.0281 \mathrm{e}-05$ & $2.5206 \mathrm{e}-05$ & $1.2619 \mathrm{e}-05$ & \\
\hline $10^{-8}$ & $1.9799 \mathrm{e}-04$ & $1.0004 \mathrm{e}-04$ & $5.0281 \mathrm{e}-05$ & $2.5206 \mathrm{e}-05$ & $1.2619 \mathrm{e}-05$ & Table 1. \\
\hline $10^{-12}$ & $1.9799 \mathrm{e}-04$ & $1.0004 \mathrm{e}-04$ & $5.0281 \mathrm{e}-05$ & $2.5206 \mathrm{e}-05$ & $1.2619 \mathrm{e}-05$ & Maximum absolute \\
\hline $10^{-16}$ & $1.9799 \mathrm{e}-04$ & $1.0004 \mathrm{e}-04$ & $5.0281 \mathrm{e}-05$ & $2.5206 \mathrm{e}-05$ & $1.2619 \mathrm{e}-05$ & $\begin{array}{l}\text { errors and rate of } \\
\text { convergence for }\end{array}$ \\
\hline $10^{-20}$ & $1.9799 \mathrm{e}-04$ & $1.0004 \mathrm{e}-04$ & $5.0281 \mathrm{e}-05$ & $2.5206 \mathrm{e}-05$ & $1.2619 \mathrm{e}-05$ & Example 6.1 at \\
\hline$E^{N}$ & $1.9799 \mathrm{e}-04$ & $1.0004 \mathrm{e}-04$ & $5.0281 \mathrm{e}-05$ & $2.5206 \mathrm{e}-05$ & $1.2619 \mathrm{e}-05$ & $\begin{array}{l}\text { different perturbation } \\
\text { parameter and number }\end{array}$ \\
\hline$R^{N}$ & 0.9849 & 0.9925 & 0.9962 & 0.9982 & & of mesh points \\
\hline
\end{tabular}

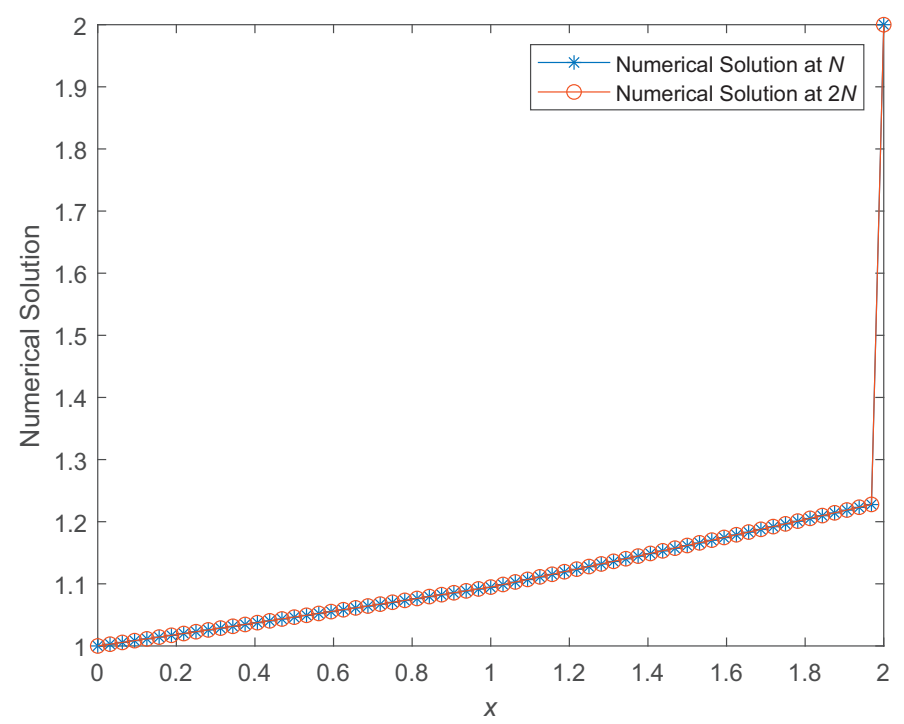

Figure 1.

The behavior of the numerical solution for Example 6.1 at $\varepsilon=10^{-12}$ and $N=32$ 


\section{AJMS \\ 28,1}

98

\section{Figure 2.}

Point wise absolute error of Example 6.1 at $\varepsilon=10^{-12}$ with

different mesh point $N$
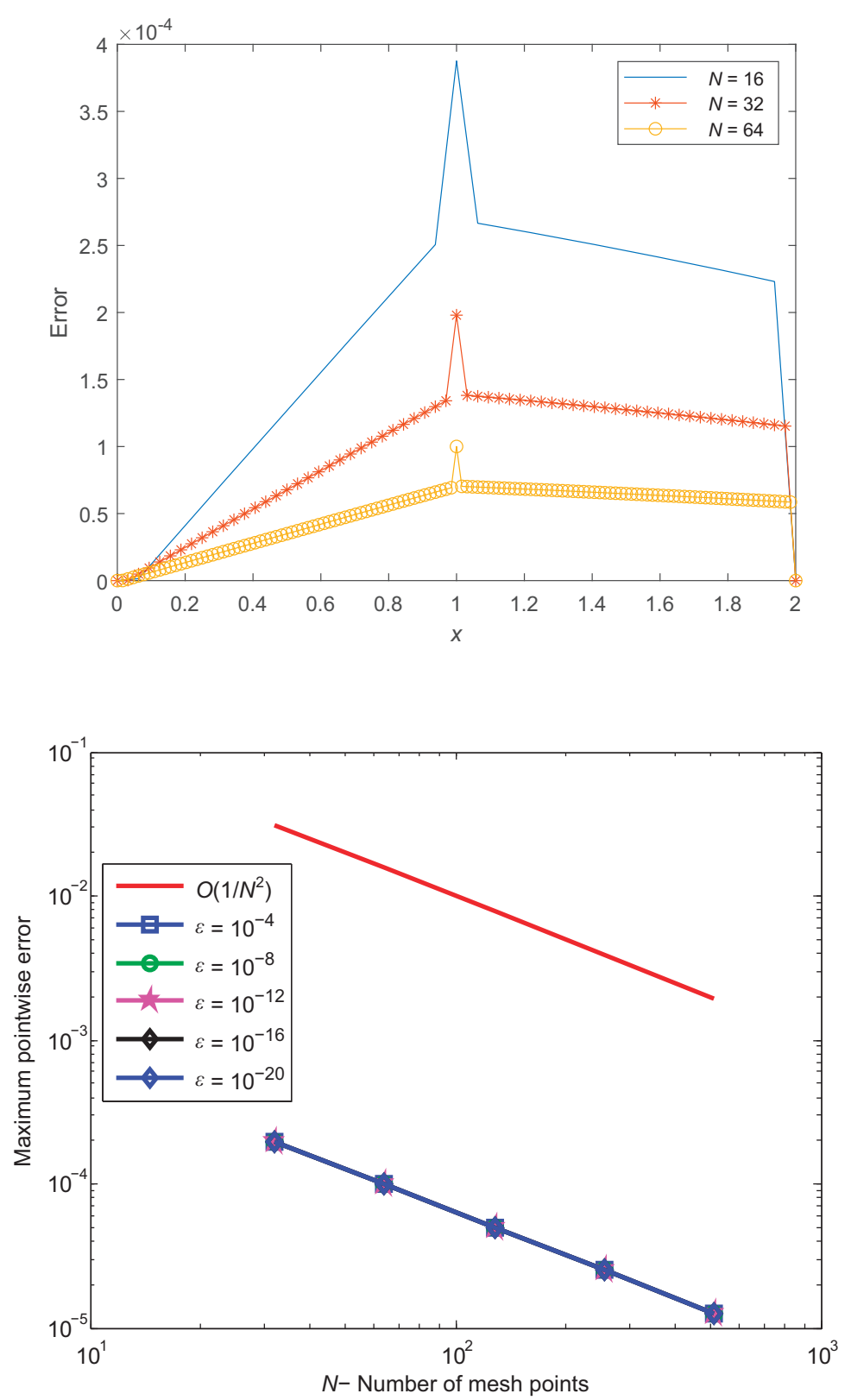

$N$ - Number of mesh points
Figure 3.

$\varepsilon$-uniform convergence with fitted operator in $\log$-log scale for Example 6.1

\section{References}

[1] Longtin A, Milton J. Complex oscillations in the human pupil light reflex with mixed and delayed feedback. Math Biosci. 1988; 90: 183-99.

[2] Glizer VY. Asymptotic analysis and Solution of a finite-horizon $H^{\infty}$ control problem for singularly perturbed linear systems with small state delay, J Optim Theory Appl. 2003; 117: 295-325. 
[3] Culshaw RV, Ruan S. A delay differential equation model of HIV infection of C D4+ T-cells, Math Biosci. 2000; 165: 27-39.

[4] Elsâ EL. ${ }^{{ }^{M}}{ }_{\text {golât }}{ }^{\mathrm{TM}} \mathrm{ts}$, Qualitative methods in mathematical analysis. American Mathematical Society. 1964; 12.

[5] Reddy YN, Soujanya GBSL, Phaneendra K. Numerical integration method for singularly perturbed delay differential equations, Int J Appl Sci Eng. 2012; 10(3): 249-61.

[6] Sirisha CL, Reddy YN. Numerical integration of singularly perturbed delay differential equations using exponential integrating factor, Math Commun. 2017; 22(2): 251-64.

[7] Gadissa G, File G. Fitted fourth order scheme for singularly perturbed delay convection-diffusion equations, Ethiopian J Edu Sci. 2019; 14(2): 102-18.

[8] Subburayan V, Ramanujam N. An initial value technique for singularly perturbed convectiondiffusion problems with a negative shift, J Optim Theor Appl. 2013; 158(1): 234-50.

[9] Geng FZ, Qian SP. A hybrid method for singularly perturbed delay boundary value problems exhibiting a right boundary layer. Bull Iranian Math Soc. 2015; 41(5): 1235-247.

[10] Kumar NS, Rao RN. A second order stabilized central difference method for singularly perturbed differential equations with a large negative shift. Diff Eq Dyn Sys. 2020: 1-18, doi: 10.1007/s12591020-00532-w.

[11] Kumar D., Kumari P. Parameter-uniform numerical treatment of singularly perturbed initialboundary value problems with large delay. Appl Numer Math. 2020; 153: 412-29.

[12] Debela HG, Duressa GF. Accelerated fitted operator finite difference method for singularly perturbed delay differential equations with non-local boundary condition. J Egy Math Soc. 2020; 28: $1-16$.

[13] Liu Z, Papageorgiou NS. Positive solutions for resonant $(p, q)$-equations with convection. Adv Nonlinear Anal. 2021; 10: 217-32.

[14] Perera K, Shivaji R, Sim I. A class of semipositone p-Laplacian problems with a critical growth reaction term. Adv Nonlinear Anal. 2020; 9: 516-25.

[15] Tang X, Chen S. Singularly perturbed Choquard equations with nonlinearity satisfying Berestycki-Lions assumptions. Adv Nonlinear Anal. 2020; 9: 413-37.

[16] Jiang S, Liang L, Sun M, Su F., Uniform high-order convergence of multiscale finite element computation on a graded recursion for singular perturbation. Electron Res Arch. 2020; 28: 935-49.

[17] Varma VD, Chamakuri N, Nadupuri SK. Discontinuous Galerkin solution of the convectiondiffusion-reaction equations in fluidized beds. Appl Numer Math. 2020; 153: 188-201.

[18] O'Malley RE. Singular perturbation methods for ordinary differential equations. New York: Springe, 1991; 89: 8-225.

[19] Siraj MK, Duressa GF, Bullo TA. Fourth-order stable central difference with Richardson extrapolation method for second-order self-adjoint singularly perturbed boundary value problems. J Egyp Math Soc. 2019; 27(1): 50.

[20] Smith GD. Numerical solution of partial differential equations: finite difference methods. New York: Oxford university press. 1985.

\section{Corresponding author}

Habtamu Garoma Debela can be contacted at: habte200@gmail.com

For instructions on how to order reprints of this article, please visit our website:

www.emeraldgrouppublishing.com/licensing/reprints.htm

Or contact us for further details: permissions@emeraldinsight.com 